\title{
CONSTRUCTIVE GEOMETRY EDUCATION BY CONTEMPORARY TECHNOLOGIES
}

\begin{abstract}
A B S T R A C T
Contemporary technological development (CAD/CAM/CAE, VR, AR, MR) made conventional methods of Descriptive and Constructive Geometry uncompleted. Application and use of new technologies in Constructive geometry requires educational process with the aim to have complete knowledge of all fields that belong to this area. The aspect and results research shows that knowledge acquired in this way by using new technology, develops students' skills that are very important in senior years of studies, particularly in the field of engineering design. Interactive dynamic 3D geometry could not be achieved by conventional ways of studying. The use of modern technology should enable expanding the fields of research as well as preservation of the theoretical knowledge of descriptive geometry.
\end{abstract}




\section{INTRODUCTION}

This aspect and results research deals with the changes in the way of teaching Constructive geometry and gives the direction of transforming the course to the contemporary requirements [11]. In order to liaise the traditional and contemporary constructive geometry approaches, used in the teaching process, a large number of powerful computer programs have been developed that are distinguished by the ease of use both in preprocessing and post-processing stage. Working in a virtual environment is a new potential for the study of Constructive geometry.

Traditionally, the Constructive geometry curriculum was based on Descriptive geometry that allowed the design of $3 \mathrm{D}$ objects in $2 \mathrm{D}$ using special constructive procedures.

Computers have changed teaching of Constructive geometry in many ways. Using contemporary technologies provides the constructive geometry education in the digital era in the field of architecture, engineering and art. Basic engineering - architectural activity is to make a synthesis of available knowledge, skills and technologies to define a particular object by the design. Conventional visualization tools should be extended to achieve a dynamic 3D geometry.

Using 3D CAD increases and expands the capabilities of conventional Constructive geometry. Certainly one of the most effective ways to train students in the constructive geometry by modeling and visualization of the objects in different spaces varying in dimensions and geometries, using CAD technology and virtual reality provides insight into the constructed objects from all sides as well as research in space, which is interactive, so this dynamic component provides excellent training of the spatial abilities of the users.

The aim of this paper is to introduce the use of contemporary technology in teaching the Constructive geometry in order to acquire the knowledge that could later be used in professional courses.

\section{TEACHING CONSTRUCTIVE GEOMETRY AT THE ENGINEERING FACULTIES OF THE UNIVERSITY OF BELGRADE}

The rapid development of CAD software made classical methods of descriptive geometry incomplete. However, this discipline is very important 
for the development of spatial intuition. In order to liaise the classical and modern approaches, software packages used in the teaching process have been developed.

Descriptive geometry is a classical geometric discipline that allows one to design three-dimensional objects in two dimensions using special constructive procedures. Constructive techniques that are applied are important for architects, engineers, designers, artists and so on.

In the recent decades CAD software has become the standard for engineers and designers. Since CAD software is used in Descriptive Geometry the practical knowledge of descriptive geometry has became insufficient. However, teaching descriptive geometry is profoundly linked to spatial intuition (Fig. 1). Therefore, the theory of descriptive geometry should be retained in the curriculum [5]. The way of harmonizing traditional and modern is merging the modern graphics software with the conventional method of teaching descriptive geometry. Graphic software in use in teaching is important not only for teaching descriptive geometry, but also for many other areas.

The conventional method of teaching descriptive geometry means that the professor uses the blackboard, a compass, a ruler, and the book as major tools. However, this equipment is not sufficient for presenting $3 \mathrm{D}$ objects and their relations (Fig. 2). Static drawings of 3D objects on the blackboard or on paper does not carry complete information describing the object. In order to fully understand the drawings of $3 \mathrm{D}$ objects they must be supported by additional text and the intuition of the user. Interactive 3D models show the spatial relationships more clearly hence allowing the user to view the object from different positions. Also, by using dynamic 3D geometry the important details in the structure (Fig. 3) can be perceived.

Solving problems in Descriptive Geometry is usually divided into several geometric steps that are simple but require a good understanding of space. By using CAD layers it helps showing relevant pictures in sequences of the structure step by step and hiding parts of the drawings that are less important. In order to develop students' spatial intuition it is useful to have an interactive tool for 3D presentation of spatial objects. The problem of the conventional methods is imprecision. By using computer software, this problem has been eliminated (Fig. 3). 

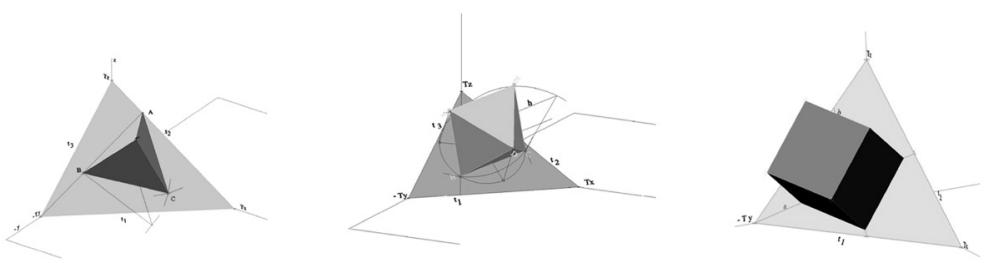

Figure 1 AutoCAD 3D models
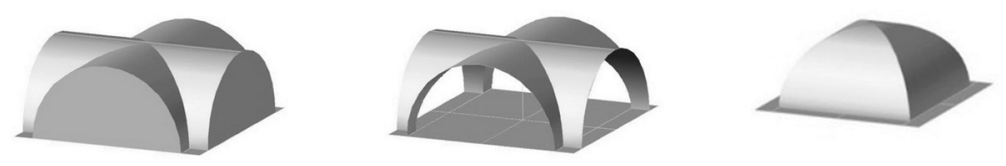

Figure 2 Boolean 3D models
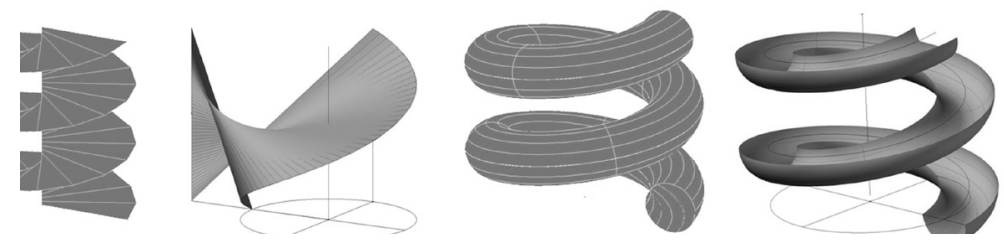

Figure 3 Surfaces 3D models
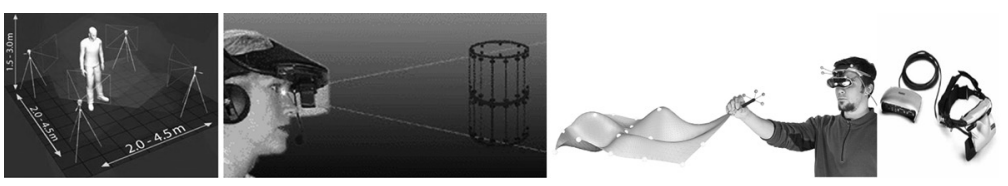

Figure 4 AR lab with HMD
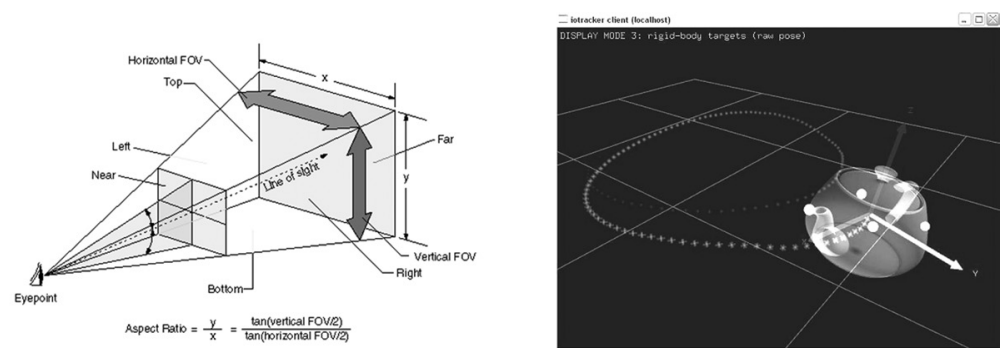

Figure 5 FOV and FOR and pose estimation 6-DOF 
According to that a variety of shapes that could be obtained from traditional geometric methods rather than limited current computer technology have brought about a real geometric revolution. We are faced with dramatic changes. Technological tools at our disposal appear to be unlimited. However, the potential of modern technology, especially in our environment, does not keep step with the development of geometry education. Thus the most important task is to harmonize technological capabilities and real working knowledge of the new geometric methods. To a large extent, structural and technological capabilities have set new challenges for engineers and designers and professions alike. We are confident that these challenges could be met with a reliable understanding and knowledge of geometry.

\section{DYNAMIC CONSTRUCTIVE GEOMETRY TEACHING}

Inclusion of CAD technology as a didactic teaching methods of constructive geometry is still required, especially the use of different 3D applications, animation and simulation methods. CAD software is a contemporary tool for the presentation of descriptive and constructive - spatial geometry. In this way, the process of learning is easier and offers great opportunities for further development of spatial intelligence [6].

To understand the potential of constructive geometry and its area of application, which is part of many advanced educational programs in technical studies around the world, the use of dynamic geometry software helps learning basic geometry of space in a visual and interactive way. Dynamic geometry allows the study of traits or characteristics of geometry in motion.

By using this tool the examples have been developed that enable teachers and students to intuitively explore interesting properties of geometric elements, objects and to visualize their relationships (Fig. 4).

The main advantage of using VR technology is that students can see 3D objects that cannot be constructed by using traditional methods.

In relation to the evaluation and observations of direct work in 3D space, complex spatial problems and spatial relationships could be understood better and faster than by traditional methods.

Currently the computer could not record and store information in the same way as the constructed geometric object appears. The software is able to do quick 
reconstruction after changing certain parameters. This is the key concept of dynamic geometry: select the item, move it and now see how this will change the structure. Movement ability is a fundamental improvement over drawing on paper or static CAD models.

Adapting traditional contents to new media is certainly a challenge but in some cases, VR technology allows one to introduce teaching of new contents. The example of that is that $3 \mathrm{D}$ dynamic geometry is impossible to teach in a traditional way - by using paper and a pencil.

The key to achieve improvement of teaching is to find the ways of taking the advantage of the new available technologies. Considering that many of the technological problems were solved in previous years and VR technology has become mature, it is more and more challenging to deal with VR didactics and pedagogy.

The four key elements in the experience of VR are: Virtual world, Immersion, Sensory feedback and Interactivity. Virtual world: an imaginary space often manifested through a medium, it is the space that exists in the ideas of the author and a description of a collection of objects in space with the rules governing relations between those objects. Immersion: The user must be immersed in another, alternative reality, to have perception of an alternative world, or the normal world from a different angle. The sense of immersion is that the user in that environment could be purely in mental terms, or it can be achieved by physical means. The physical immersion is a characteristic that defines a virtual reality. Concretation is probably the aim of the majority of media creators. Sensor feedback: Unlike traditional media, VR allows the participants to choose the point of positioning their body and to influence the events in the virtual world. The VR system provides direct sensory feedback to participants on the basis of their physical location. Interactivity: For virtual reality, for the authentic look which should respond to the user actions, it must be interactive [2].

Collaborative environment means multiple users to interact in a virtual world that allows interaction between participants. Collaboration in the VR environment means more participants that can be present at the same time and who could all participate in the work. Virtual reality is a medium that consists of interactive computer simulations in the sense that they share one or more senses, giving a sense of immersion or the presence in the simulation (virtual world). 
Virtual environment is a virtual world, or an instance of the virtual world presented through interactive media such as virtual reality.

Avatar - the term comes from India and means the incarnation of God Vishnu used in applications where a real person controls his/her visual clone which does not have to resemble the original. Avatar is a virtual object that is used to display the participants in the virtual world. The visual could be any shape or object included by the user [10].

\section{D user interface}

Visual displays are the information to the human receptive system. Display devices require a computer system to generate digital content that is translated into a perceptible form.

Characteristics of visual display: The field of regard and the field of view (FOR $=$ Field Of Regard and FOV = Field Of View), spatial resolution, the geometry of the screen, the light transmission mechanism, refreshing, ergonomics and other features include brightness, color contrast and gamma correction.

\section{Field of regard and field of view}

FOR $=$ Field Of Regard refers to the amount of physical space that surrounds the user in which visual images are displayed. FOR was measured in degrees of visual angle. For example if we have a cylindrical display in which the user stands, the display will have horizontal FOR angle of $360^{\circ}$ (Fig. 5).
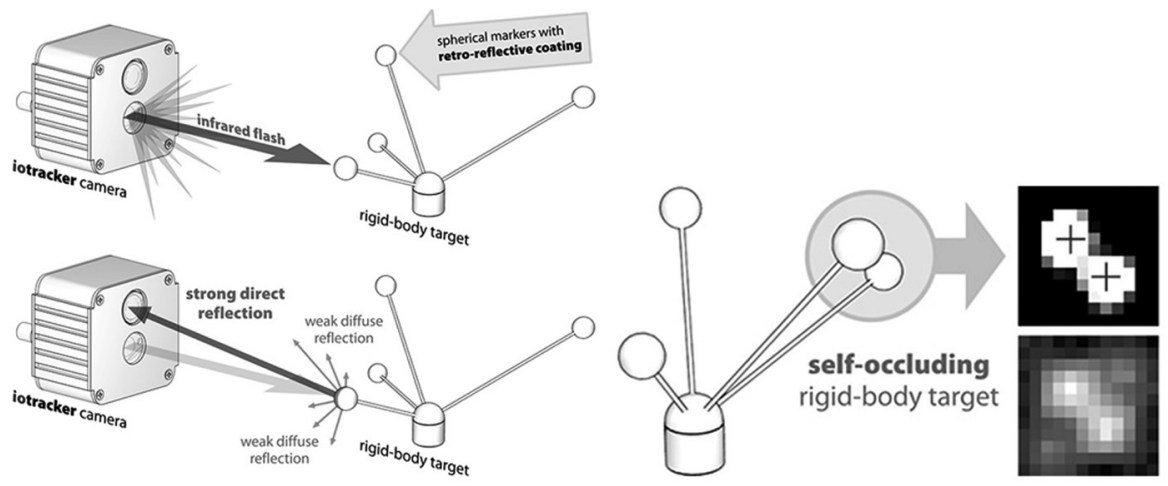
$\mathrm{FOV}=$ Field Of View refers to the maximum number of degrees of visual angle, which can be currently seen on the display. For example, a large, flat screen horizontal angle (FOV) can be from $80^{\circ}$ to $120^{\circ}$ depending on the viewing position in relation to the screen, because it varies with the removal of the FOV to the screen [2].

\section{Degree of freedom}

DOF - Degree of freedom is a geometrical definition of the freedom of movement (displacement) along the axis in space or around the axis of rotation in space. In 3D space there are six degrees of freedom: three degrees of movement in $\mathrm{x}, \mathrm{y}$ and $\mathrm{z}$ direction and three degrees of rotation around the $\mathrm{x}, \mathrm{y}$ and $\mathrm{z}$ axes (Fig. 5).

\section{Principles of Iotracker System}

Iotracker system uses passive targets of a rigid body made of retro-reflective spherical markers. Markers with a special coating reflect infrared light emitted by iotracker camera's built in LED strobe back to the sensor (Fig. 6).

Iotracker software makes advanced image processing algorithms to calculate the projected centers of each marker in each images of the camera (Fig. 6).

3D location of each marker is obtained through geometric triangulation.

Iotracker software identifies the pre-calibrated objectives of a rigid body and calculates their position and orientation (6-degrees of freedom) (Fig. 7).
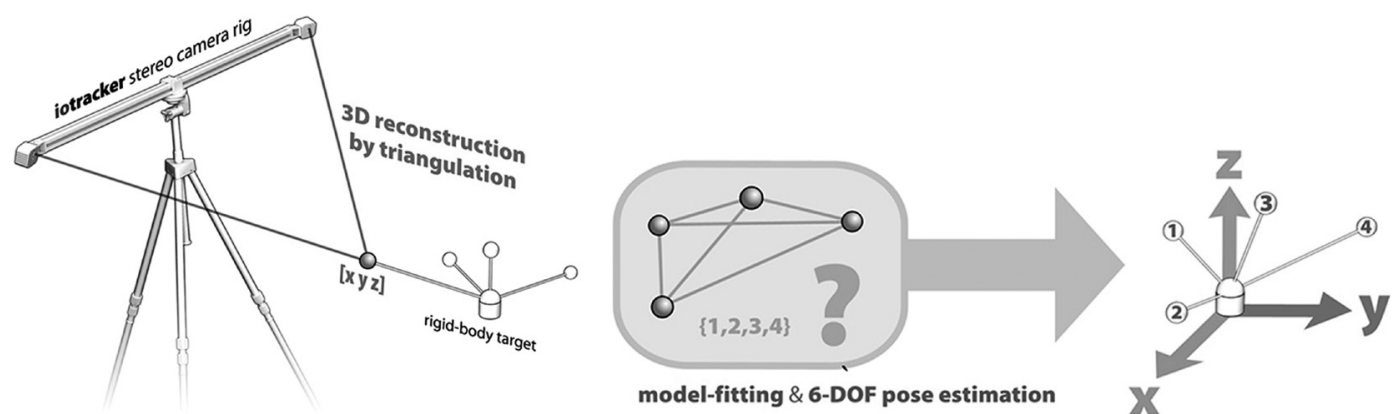
The aim of the objective measurement of optical technology is to calculate the exact position and orientation of tools, objects or persons in a predefined coordinate system. Rotating optical trackers usually use more 2D images sensors (cameras) to detect active-infrared emission or passive - retro-reflective label fixed to some interaction devices. Based on information obtained from multiple cameras, the system is able to calculate the position of each marker through geometric triangulation. When more than two markers are grouped together to form a rigid body, it becomes possible to determine the orientation of the target, collecting a total of six degrees of freedom (6-DOF).

\section{Iotracker system display}

Typical iotracker system (see Figure below) consists of four to eight iotracker cameras, one iotracker synchronization unit, workstations - computer with iotracker software and several rigid body markers fixed for different interactive devices [13].

Iotracker camera could be mounted on wall or on tripod. These closures are synchronized to trigger-pulse signal sent by the Centre for iotracker synchronization unit via coaxial cable. Each camera is connected to digital video monitoring workstation. Iotracker software running on the workstation calculates the position and orientation of each rigid body marker in real time and transmits the result of measurement with 6-degrees of freedom on the reported client machines through eternet network [13].

\section{AUGMENTED AND MIXED REALITY}

Augmented reality is a kind of virtual reality in which additional information exists, imperceptible to human senses, and which is noticeable and registered in the enlarged depiction of reality with the physical world. Virtual reality applications are designed to combine the virtual representation with the perception of the physical world. The difference between AR and VR is that in VR the real world is completely replaced by the virtual one. Mixed reality is shown by Milgram's diagram (Fig. 8).

Since most of the available software for dynamic geometry is limited to the Euclidean plane, CONSTRUCT3D software can be used to study 3D problems. Hannes Kaufmann dynamic geometry software is implemented in VR [1]. 
CONSTRUCT3D is a three-dimensional dynamic geometry tools to construct which could be used in education at the university level. AR / augmented reality is used to provide a natural setting for face-to-face collaboration between teachers and students. The main advantage of using VR and AR is that the students can see the 3D objects that so far had to be constructed by the traditional methods (pencil and paper). By direct work in 3D space complex spatial problems and spatial relationships could be understood faster and in better way than by the traditional methods.

In the AR environment (Fig. 9), multiple users have access to the common area filled with virtual objects while the user is able to simultaneously monitor events in the real world. Because of the mixed real world the users can see each other. This approach is especially powerful for educational purposes when users are positioned so that they could use natural communication (speech, gestures, etc.). It is also possible to make a combination with VR or collaboration at a distance. Supporting natural collaboration within the domain of Constructive geometry has opened up new opportunities in the educational process.

The main advantage of using AR is that students actually see the 3D objects they used to construct using traditional methods. Working directly in 3D space complex spatial problems and spatial relationships could be understood in a better way and faster than by the traditional methods. This AR system as a medium of teaching and 3D dynamic geometry have been designed to facilitate teaching geometry. Both aspects are new in geometrical education. Work in Construct3D does not create professional 3D package for modeling but simply intuitive constructional 3D design tools as virtual environment for educational purposes (Fig. 10).

Construct3D supports generation and operation of the basic geometric types of objects: points (freely positioned in space or fixed on curves and surfaces), lines, planes, circles, ellipses, cuboids, spheres, cylinders, cones, B-Spline curve with unlimited number of control points and different degrees, interpolated B-Spline curves, NURBS surfaces to $8 \times 8$ control points and different levels, interpolated NURBS surface and surface of revolution (rotational sweep surfaces). As regards geometric operations, Boolean operations (intersection, union, difference) are embedded between the intersections of all types of $2 \mathrm{D}$ and $3 \mathrm{D}$ objects that result in sections of points, curves, planar sections of objects, a rotational sweep around the axis, and many others. Transalations, rotations and reflections are also included. 


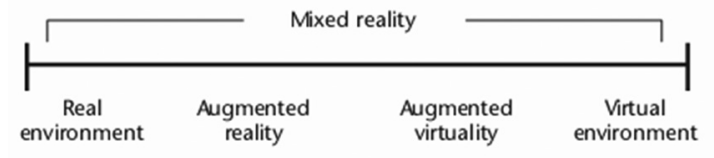

Figure 8 Milgram's reality-virtuality continuum

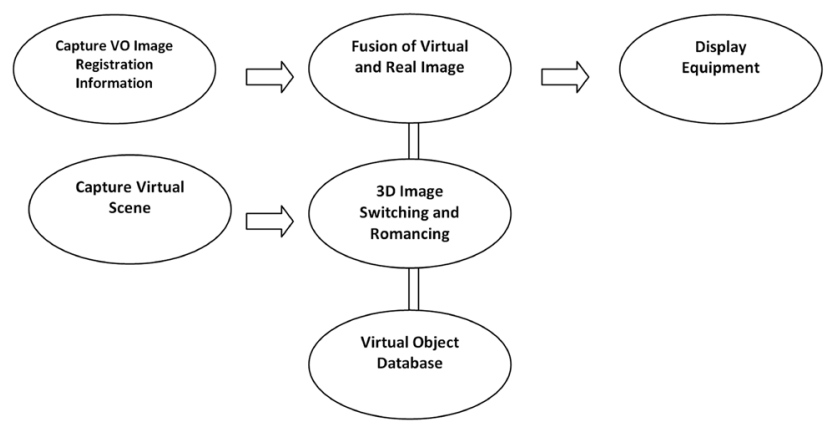

Figure 9 Basic framework of AR system
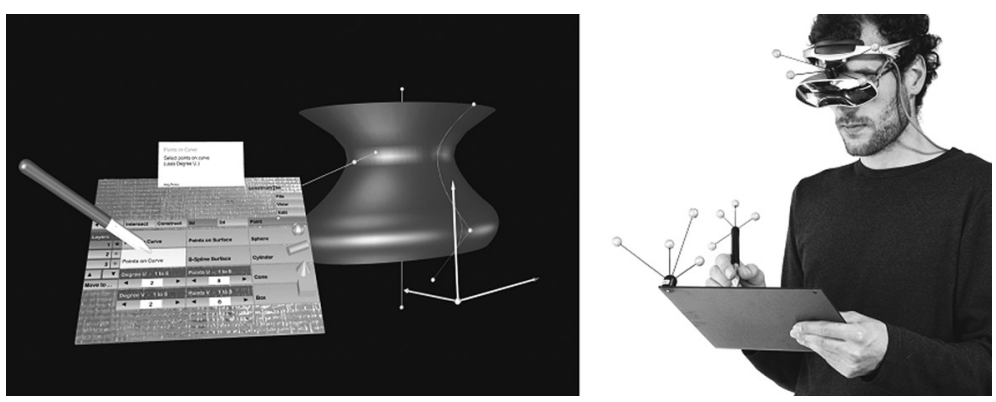

Figure 10 Student works with CONSTRUCT3D holding a pen and a wireless optical tracking panel which uses retro-reflective markers. Student's current view through HMD and visible system on tablet.

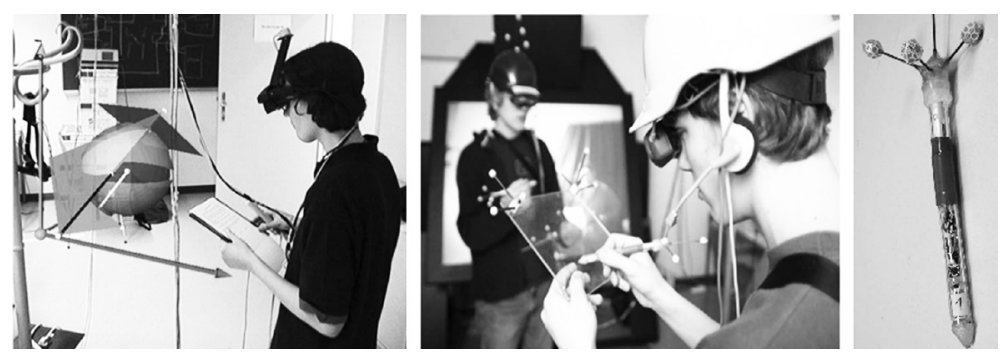

Figure 11 Volume visualization and interaction tablet and pen
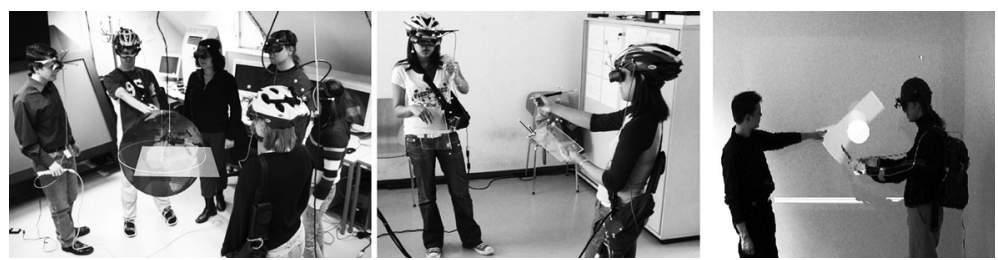

Figure 12 Volume visualization and interaction tablet and pen 
The system menu is mapped on tablet that is in user's hands and is called a personal interactive panel (PIP $=$ Personal Interactive panel). PIP allows direct incorporation of conventional 2D interface elements such as buttons, keys, etc. and the newer 3D interactive widgets. Passive tactile feedback information from physical equipment helps to guide users when interacting with PIP while presented graphics allows the equipment to be used as multifunctional tools (Fig. 11).

All construction steps are carried out by direct manipulation in 3D by using a tracking form with six degrees of freedom. In order to generate a new point the user clicks with the pen to show accurately the place in the 3D space where the item should appear. It is possible to bring a new point and a new $3 \mathrm{D}$ objects on the existing virtual model.

The basic characteristic of dynamic geometric software is that the dynamic behavior of structures could be explored by interactive individually defined geometric elements. For example, moving a point that lies on the sphere results in a change in the radius of the sphere. Then it is possible to see which parts of the structures are changing and which remain unchanged. The interdependence of geometrical structure remains unchanged. The motion enables better understanding of what is happening in construction and in geometry in general.

\section{COLLABORATIVE EDUCATIONAL AR SETUPS}

A standard setting for Construct3D supports both users to cooperate wearing stereoscopic HMD (Head Mounted Display) see through which allows them to share the common virtual space. The users interact with the system using a pen and a tablet. Both users see the same virtual objects as pens and menu systems on the panels - which enable the students and the teachers - to help another user if necessary (Fig. 12).

Other hardware settings such as desktop, based on projections, mobile and hybrid settings were tested during the development of Construct3D by Hannes Kaufmann TU Vienna. They all have pro and contra arguments, but the huge advantage of using HMD is that the setting allows users to start "walking around" geometric objects that are fixed in space. This is a unique feature of HMD settings that could be enabled using a monitor or projection screen based on hardware configuration [8]. Forming VR lab in Vienna Hannes Kaufmann calls for papers Hix and Gabbard 2002. 


\section{Experience in $A R$}

Augmented reality could be defined as a variation of the virtual environment or virtual reality as it is usually called. Virtual environment technology introduces users to completely artificial (synthetic) environment. Whilst in VR the user could not see the real world around him, on the contrary the AR allows the user to see the real world, with superimposition over or incorporation into the reality. Therefore, AR supplements the reality rather than it replaces completely. Some researchers define AR as a method that requires the use of HMD. To avoid the limitation of specific-AR technology, AR system could be defined with the following three effects. They are: a combination of real and virtual, interactive real-time registration in 3D. This definition allows other technologies to engage, other than HMD, and retains the essential components of AR.

Briefly augmented reality includes the expansion - the enrichment of the real world with complementary virtual one.

Through discussions with the teachers three key directions Construct 3D have been identified.

The first and the most important one - the construction of dynamic 3D geometry is a major advantage. 3D dynamic geometry could be realized with a pen and paper or with a static program for modeling. Interaction with geometric objects provides support through research learning.

Students could actively change positions around the objects. Virtual environment is designed by spatial relationship between the virtual objects and the body of the student.

The teachers highlight in particular the strength of visualization of the abstract geometric problems. Therefore, the ideal contents of this educational environment is AR geometric study of dynamical properties, to encourage modification, and visualization of abstract geometric problems.

For the purpose of teaching specific curriculum contents teachers usually choose the teaching tool (a medium for learning) that is best tailored for learning specific contents. According to positive world experience Construct3D should be integrated into education for the courses that require a 3D dynamic geometry or visualization of abstract problems. In addition, there are areas that are difficult to perform with other teaching aids. Problems that might well be 
solved in Construct3D could be difficult to implement or impossible to solve with usual approaches. There are also tasks which are convenient for oldfashioned 2D 'paper \& pen' solution and could be impractical in Costruct3D.

The mediums for education are different and it is logical that the teaching contents should be adjusted accordingly. The advantages of the AR setting are numerous. The users see their bodies and arms and the effects of their actions while working. Constructive process physically involves students and is similar to manual labor more than the traditional computer operations. A spatial relationship between the user's bodies and geometry structures in 3D space is established. These are key factors in the potential success of using AR in geometry teaching.

Costruct3D has been developed to the point where it can be productively used for educational purposes. It has a wide range of useful features that use dynamic functionality to generate simple geometric forms in a short time, for both teachers and students. This tool allows a new way of education.

Dynamic 3D structures and visualization capabilities are fundamentally strengthened by using AR. Designed contents must relate to these findings. It is obvious that a simple, introductory geometry could do tasks faster and easier by drawing by hand or in CAD software, saving lots of money for a set of VR hardware. The challenge is how to design interesting contents and match it with an adequate 3D dynamic geometry application. Creation of new contents for 3D dynamic geometry is a new challenge for the teachers, which in turn broadens the knowledge of geometry. Dynamic 3D geometry is both the new field of research and the one which is still in development. The question remains why successful and well-developed Construct3D is not yet used in teaching. After many discussions and conversations with teachers and students three major obstacles were identified. They apply not only to Construct3D but to a broader range of VR educational tools.

Three major obstacles are: the cost of hardware, still limited number of users and the technical complexity [7].

The current VR / AR hardware is expensive mainly because there is no mass market for VR / AR equipment. It is very unlikely that an average student could afford such impressive equipment favored by teachers and students alike. Accurately tracking the location and orientation of multiple users and all their equipment is the most expensive part of the system. The main 
reason why developers are encouraged to make an inexpensive optical tracking system - iotracker - is to minimize the cost of tracking. The aim is to integrate Construct3D in university education. Prices are still high in relation to available funds, but changes have lately been seen in the market. Most of the application tools discussed here allow only a small number of users. That alone reduces the access of many to this advanced technology. Integration into regular classes becomes complicated if only a small number of students have opportunity to use the advanced technology. Pedagogical concepts are needed for the integration of high technology as an important way that all students could benefit from. Spare option is the use of VR technology in special courses with only a few attendees.

Even if hardware were inexpensive the enthusiasts declined using Construct3 $\mathrm{D}$ because of the space and setup time requirements and because of technical complexity. Technical setting consists of several components that are prone to error and require maintenance. Even the standard computer labs, with the usual equipment: monitors, mice and keyboards, require regular maintenance and operation. No matter how simple additional technology, it introduces new complexities. For a system maintenance consisting of added components such as: tracking system, interaction device, additional display devices, wireless communications the additional staff is required (at least to work several hours a week).

Stability and reliability are important criteria for educational software in general. Accurate results were absolutely important and if software should break down, the students could easily lose their motivation.

\section{THE APPLICATION OF VR}

The progress in design and growing powerful computer processors, allow expanded use of augmented (mixed) reality demonstration and testing on construction systems and space structures. Space structures are most often constructed from a number of components similar in size and shape (typically cylindrical rods and the spherical connection). Therefore, it is relatively easy to connect parts wrongly without noticing. Tracking users and objects in the space of mixed reality systems allows the users to have the visual information related to the physical environment. 

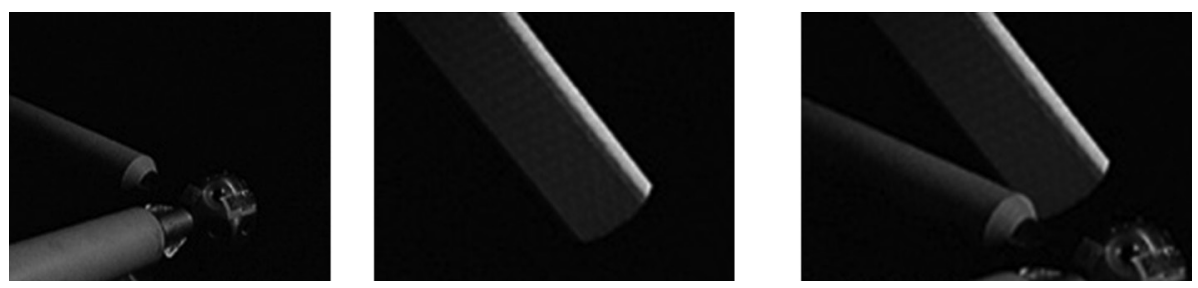

Figure 13 Only real struts / Only virtual strut / Mixed AR - real and virtual struts
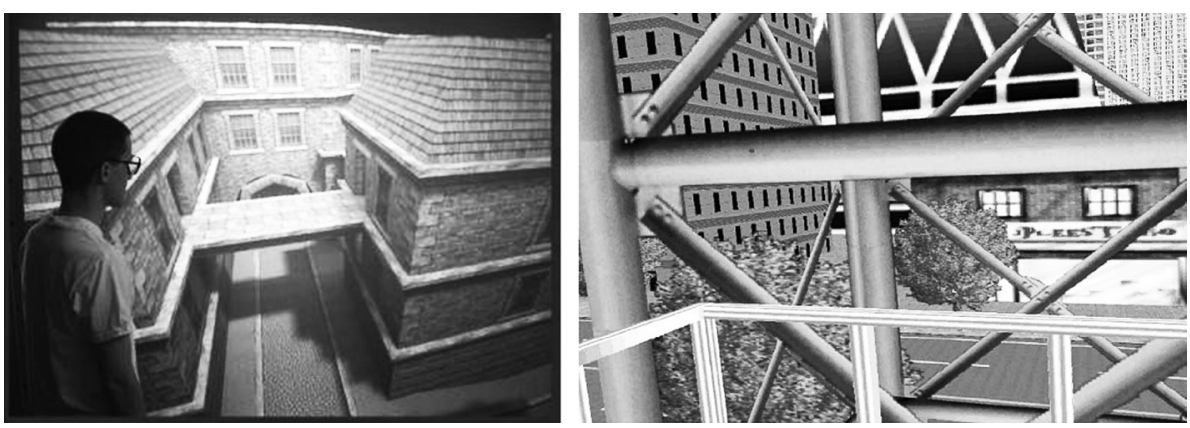

Figure 14 Application of AR in Architecture

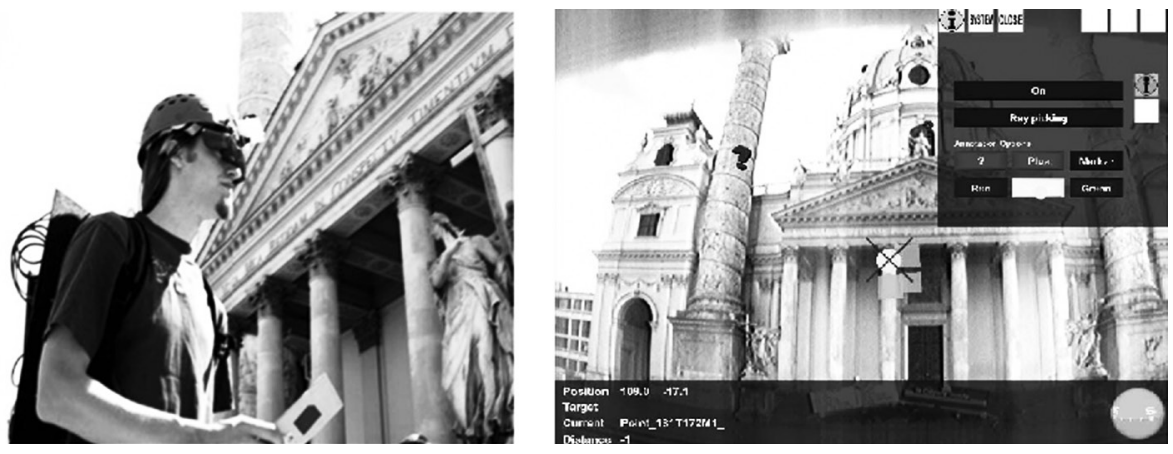

Figure 15 Mobile Augmented Reality 
Augmented (mixed) reality system, designed to lead a person to demonstrate and test work on the structure could help to ensure that every part of the set is correctly connected (Fig. 13).

This AR (mixed) system allows the user to see the natural environment other than that normally seen [4].

Direct application of Constructive geometry is in the space structure education. Further use is in visualization of complex structures (for example, CAD model, settings with large amounts of data, etc.) VR has significant benefits in training, especially if they are expensive and dangerous. Training of surgical procedures, where surgeon practices surgery on virtual organs. Flight simulation, assembly simulation, the simulation of maintenance, etc.. VR therapy and rehabilitation. Virtual prototypes [14]. VR can be used to build virtual museums, archaeological sites, interactive art, etc. VR entertainment (e.g. games and sports industry). Training and Education (Fig. 14). In addition to the numerous possibilities for application of these approaches, the application of AR in Construction systems and Space structures is particularly important [15].

\section{THE FUTURE OF VR}

The number of universities that offer courses in VR will be increased. At this moment it is hardware that limits the number of students in the group to a small number. VR will be primarily used for multidisciplinary solutions. Students use VR technology in their specific fields of study rather than learn about VR only as technology. In all areas of VR and AR technologies the "faster, better and cheaper" is a must.

Development trends are as follows:

- Less stress / In the future the user will use the screens that are no larger than sunglasses, no strings attached, to the visual and sound immersion medium.

- Increased use of augmented reality (AR) / AR applications will improve tracking and other input technologies. New technologies would allow data collection in the real world, which would spread out the areas in which AR could be successfully applied.

- VR at home - Application at home (i.e. games). Reduction of costs for most technologies.

- Machine-ready for VR - Computer systems are delivered pre-equipped with basic hardware and software components to run VR applications.

- Mobile augmented reality (Fig. 15) 


\section{CONCLUSION}

Modern applications of computer graphics could create an environment where virtual reality can interact with objects in three-dimensional scenes. Specialized hardware devices allow the user to see 3D objects and the associated effects as well as to manipulate the scenes. Animation in a virtual reality environment allows practicing complicated operations and requires analysis of different configurations and layouts within a space. Using virtual reality systems, designers and others could move through space and could examine the interaction between objects in different views. Architects could walk through a designed building from room to room to look at everything in order to identify possible deficiencies and resolve problems. The method advantages are in savings in products development before entering the serial production -the deficiencies could be spotted in time without investing money in the models or pilot series. Virtual reality allows a designer to understand the objects at the scene and move them, also move the scene in order to come up with more effective solutions. Virtual models are passive tools for visualization. They can inspire new geometric topics. When students are actively involved in specific virtual models development, they would be able to figure out their geometric properties and find the facts along the way. In this paper we have tried to show how Constructive geometry education at an engineering faculty should look like using this new technology (AR, VR and MR). In our opinion, a good knowledge of the basic principles of Constructive geometry is crucial in working with CAD packages. Students could be ready for this new technology only if possessing sufficient traditional knowledge of geometry.

Geometry is the basic tool while the technologies (VR, CAD) are only derived tools [3]. By establishing simple and clear way to the basic of the complex geometry has bene conceived in order to facilitate the students to use their geometric knowledge for further work. However, no doubt that students will definitively enhance their own creativity by using this new technology. The applications presented are all interesting in their own way [12]. They provide insight into the potential that allows the use of VR for geometry education. They do not use the traditional and present contents in a new form but rather try to find innovative ways of teaching geometry in a new manner. With the introduction of technology into the curriculum - such as graphic calculators in the recent past - students would grasp what previously stayed beyond their reach.

The examples presented provide guidance on how VR can be used for special target groups such as hearing, the special geometry domains such as dynamic geometry, and they show how contents could be adapted to become real, visible 
and understandable, even in the cases of abstract geometry. They all have one thing in common: the extremely motivating way it was presented which would not be forgotten. Even if there are difficulties which prevent the use of VR in teaching geometry, the contemporary technology is fascinating for the students and that should not be ignored. It would be irrational not to use new technology to raise the level of geometry skills of the new generations of students. Writing and oral skills of engineers fuse into own language called drawing [9].The engineers will continue to develop future visual communication and expand their interdisciplinary skills but drawing would be the main language tool for engineers worldwide. The superiority of contemporary technology has a crucial implication for teaching Constructive geometry and engineering graphics. The connection between geometry and contemporary technologies is substantial. BT/RESEARCH/SPACE-FR/testbed.html 
Kaufmann, Hannes., and Schmalstieg, Dieter. "Mathematics and geometry education with collaborative augmented reality". Computers \& Graphics, 27(3), (2003): 339-345.

Ostrogonac-Seserko, Ruza and others "Visual Communication Curricula for the Global Engineers" KOG Journal of Croatian Society for Geometry and Graphics, no. 5 (2002.): 6571.

Sperka, Martin: "Geometry in Graphics and Multimedia Courses at theFIIT STU Bratislava" Slovak Journal for Geometry and Graphics, volume 1, no. 1, (2004): 55-66.

Stachel, Helmuth: "Descriptive Geometry in today's engineering curriculum”. Transactions of FAMENA 29/2 (2005): 35-44.

Stanney, Kay M., Handbook of Virtual Environments Design, Implementation, and Applications: Lawrence Erlbaum Associates, 2002.

TU Vienna http://www.iotracker.com/

University of Columbia. "Augmented Reality in Architectural Construction, Inspection, and Renovation" University of Columbia, http://www.columbia.edu/cu/gsapp/BT/RESEARCH/ PAPERS/ar-asce.html

Zloković, Đorđe., Koordinirani sistem konstrukcija, Građevinska knjiga, Beograd, 1969.

N.B.

Special thanks for material used in this paper to professor Hannes Kaufmann from TU Vienna. Authors are suppored by the project TP 36008 of the Serbian Ministry of Science and Technological Development 